\title{
The Design of MLX90621 Based Intelligent Lighting Control System
}

\author{
Hui-jiao Wang ${ }^{*}$, Meng-meng Liu and Cong-cong Shi \\ School of Mechanical Engineering and Electronic Information, China University of \\ Geosciences, Wuhan, China \\ ${ }^{*}$ Corresponding author 1332535134@qq.com 13036124462
}

Keywords: MLX90621 Infrared array sensor, DMX512 protocol, WIFI module, STM32.

\begin{abstract}
Intelligent light control are expected to be used in many aspects ranging from public places to private house. Combining light control with people's position offers new research interests. This paper presents an interactive intelligent lighting control system, which can light the lamps by people's position. The system uses MLX90621 sensor to collect the human body temperature data, combined with microcontroller module and average threshold method to analyze multiple pixel data in real time. Then the object's location can be accurately detected. At the same time the lighting control module receives the information by WIFI module, then it uses RS485 interface to drive DMX module to control the Philips lamps. The proposed system has been verified on $4 \times 4$ lamps matrix test platform and achieves dynamic effect that the lamps are lighted when people's position change.
\end{abstract}

\section{Introduction}

With the rapid development of science and technology, the study of new-type environmental intelligent lighting control system with multi-function becomes more and more important [1]. New-type lighting control system, which takes energy saving into consideration, should satisfy peoples increasing demand for more comfortable and convenient lighting control system [2,3]. This intelligent lighting control system lights the lamps by people's movement. And its capacity of delivering the state data of lamps to smart-phone by WIFI module makes intelligence into reality [4-6].

\section{Hardware Design}

The hardware of interactive intelligent lighting control system based on MLX90621 sensor is composed of two main module: lighting control module and sensor module. In lighting control module, STM32F207 is used as main chip. AMS1117-3.3 is used as power chip. DMX adopts RS485 transform interface to drive Philips matrix lamps [7]. In sensor module, STM32F103 is served as the main control chip, power chip is the same as the above module, and MLX90621 is adopted as the sensor to collect person location information. In addition, the WIFI module based on LN13UA06 to implement the sending and receiving of data. Figure 1 shows the system block diagram. 


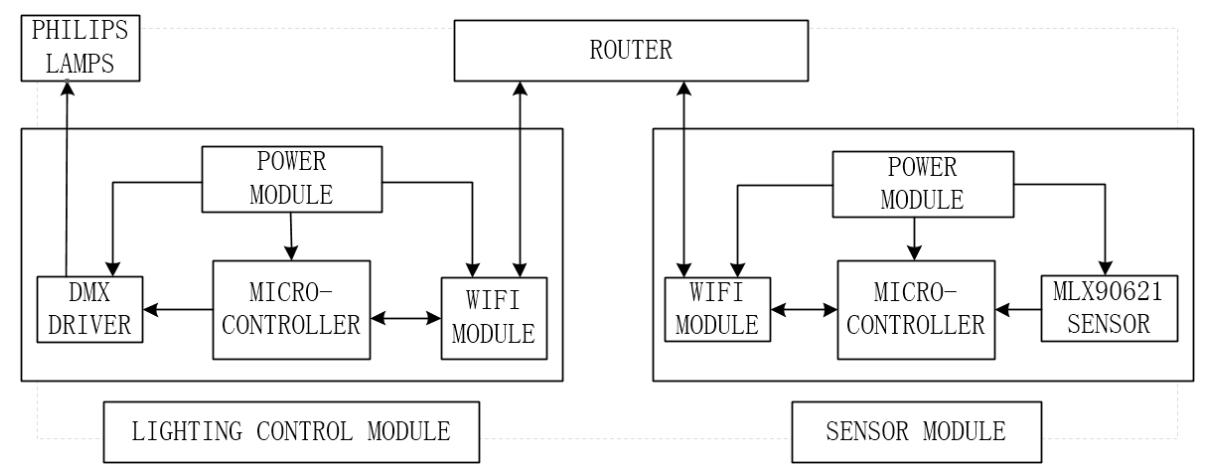

Figure 1. The system block diagram

\section{Sensor Module}

The sensor module consists of STM32F103 micro-controller, MLX90621, power module and WIFI module. STM32F103 is used as the main control chip of the module. Power module adopts double power design, which are that master USB output $5 \mathrm{~V}$ or $12 \mathrm{~V}$ get through the $5 \mathrm{~V}$ and $3.3 \mathrm{~V}$ voltage chip to supply other peripherals. WIFI module is set as the AP mode. MLX90621 infrared sensor collects the temperature of the human body through the IIC interface, and transmit it to the micro-controller for analysis and processing, then micro-controller communicate with WIFI through the serial port. Figure 2 shows the block diagram of sensor module [8].
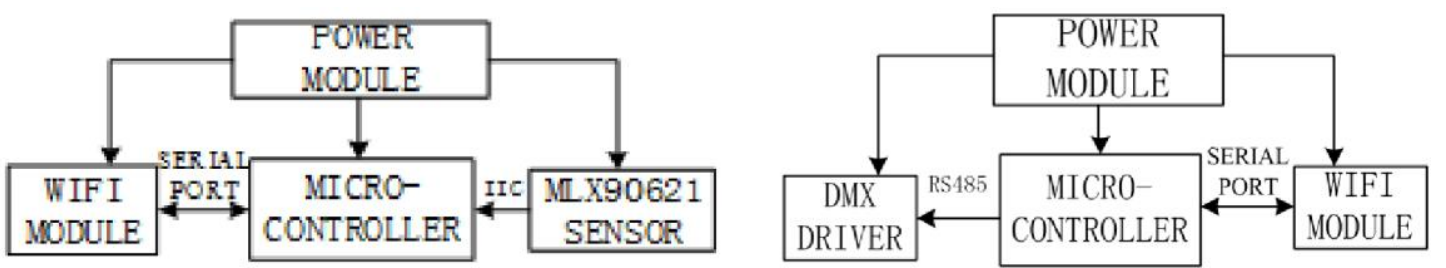

Figure 2. The block diagram of sensor module Figure 3. The block diagram of lighting control module

\section{Lighting Control Module}

Lighting control module consist of STM32F207 micro-controller, DMX512 driver, power module and WIFI module. Peripherals drive DMX512 lamp control interface by RS485 bus port [9]. Power module use USB to output 5V stable voltage, which to supply system other peripherals. WIFI module is set as STA mode. Figure 3 shows the block diagram of lighting control module.

Figure 4 shows DMX512 communication interface circuit. It uses 3.3V low power consumption and half-duplex transceiver chip ISL3178EIBZ to design the communication interface circuit of DMX512 protocol [10]. 


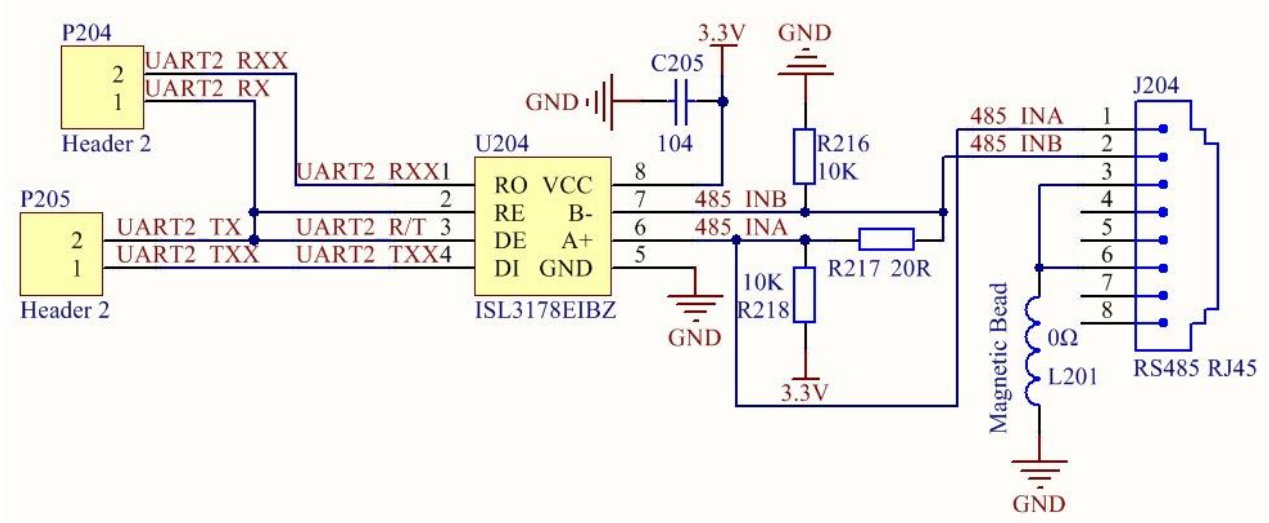

Figure 4. DMX512 communication interface circuit

\section{Locating Algorithms and System Test}

\section{Locating Algorithm}

MLX90621 detecting matrix temperature data: the MLX90621 can detect the range of a $16 \times 4$ matrix, and deliver it to micro-controller STM32. The data are processed by MATLAB, then a picture of temperature data distribution is obtained. Figure 5 shows the temperature distribution of the infrared array.
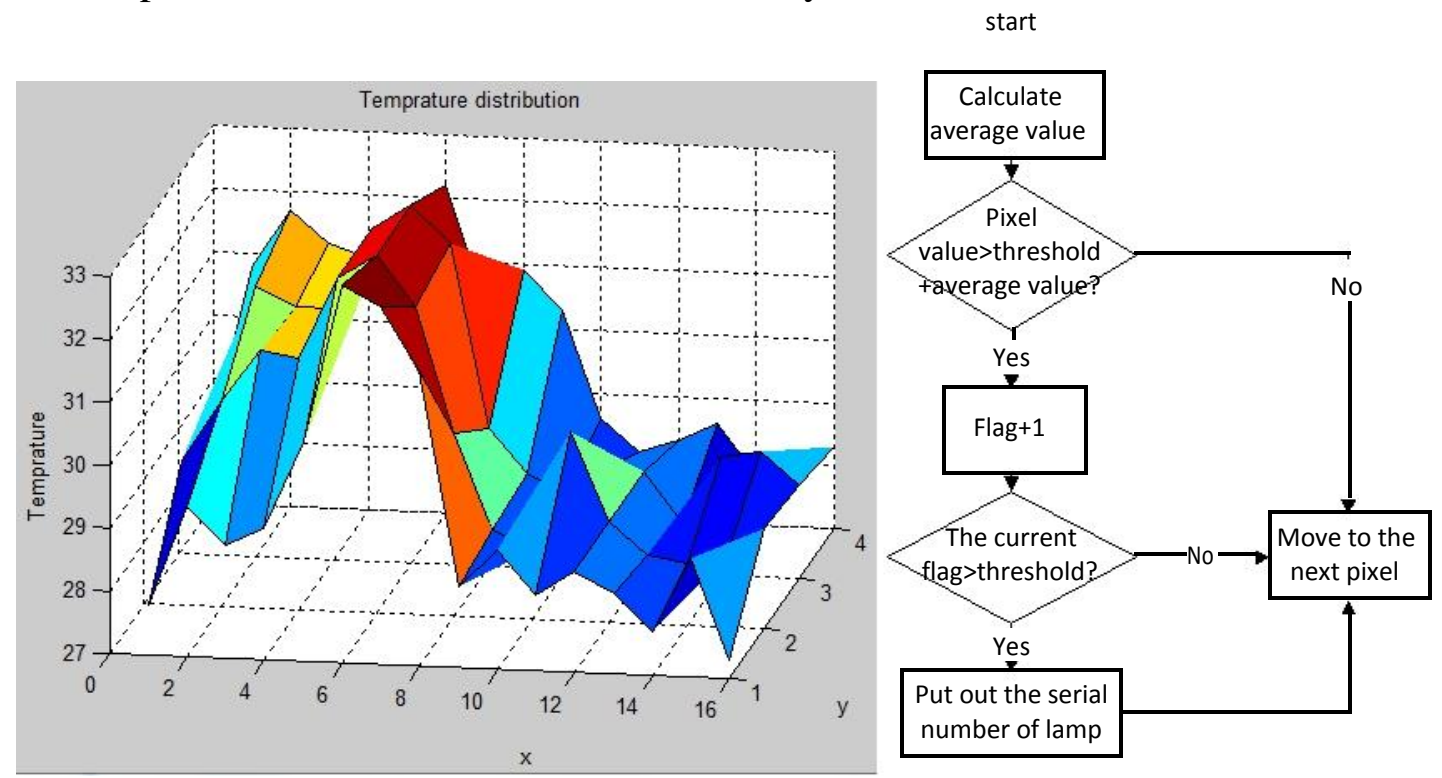

Figure 5. Temperature data distribution of infrared array Figure 6. Flow chart of locating algorithm

Locating algorithm: calculate the average value of the matrix and set a threshold. And compare the sum of the threshold and average value with the temperature of every pixel, then add 1 to the flag at the pixel which have higher temperature than the threshold. When the amount of the pixel whose flag is set as one accumulate to a relative large number, it can be assumed that a person is standing below. Figure 6 shows the flow chart of the algorithm.

\section{System Test}

Intelligent control system test: at a space of $2.3 \mathrm{~m}$ in height, $2 \mathrm{~m}$ in width and $4 \mathrm{~m}$ in length, test the system with more than one person on $4 \times 4$ lamps matrix. Figure 7 
shows the multi-people test result. Then, one of the people moves. The number 2 light is lighted with the people's moving. The number 1 light go along with the people's leaving. Figure 8 shows the test result. This test can certify that our system can work perfectly.

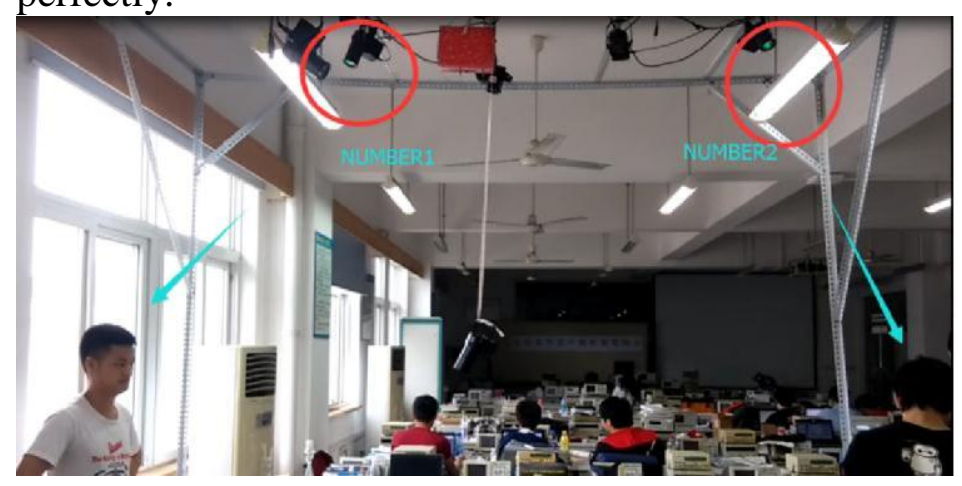

Figure 7. Multi-target test

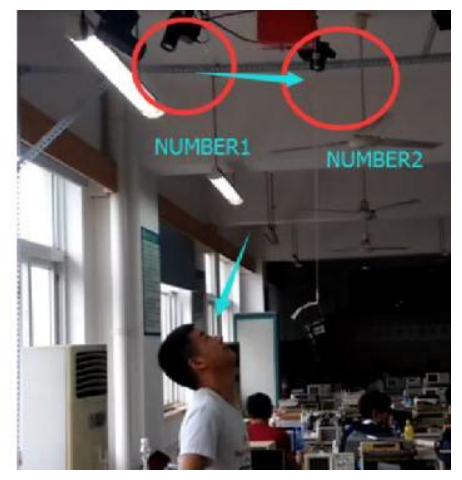

Figure 8. Moving test

Consumption of this system: the consumption of our system is $1.1 \mathrm{~W}$. Therefore, our system can realize the goal of energy saving.

\section{Conclusions}

This paper provides a comprehensive process of the design on an interactive intelligent light control system with MLX90621 sensor. This system has been verified on test platform, which includes lighting effects and the whole system consumption. First, the system can detect multiple targets and single target, then lights the lamps. Next, it achieves dynamic detection and static detection in the meantime. Last but not least, the consumption of the system realizes the goal of energy saving.

In future work, we will devote ourselves to reduce the system test misjudgment. A new fixed threshold segmentation based on histogram statistics and multi-frame sampling will be applied [11]. The method of using fixed global threshold to realize the image segmentation will reduce the misjudgment.

\section{References}

[1] Zhan-kun Yan, Research on Control System of Intelligent Lighting, (2013) In Chinese.

[2] Bayeh, Claude Ziad: British Journal of Applied Science \& Technology (2014)

Matta Sherif, Mahmud Syed Masud. An Intelligent Light Control System for Power Saving. In: IECON 2010 - 36th Annual Conference on IEEE Industrial Electronics Society, Glendale, AZ, 2010, pp. 3316- 3321.

[3] Cheng-xiang Bo. The Design and Implementation of Indoor LED Lighting System Terminal Based on WIFI (2013) In Chinese.

[4] Tanaka Junichi, Imamoto Hiroshi, Seki Tomonori, Low Power Wireless Human Detector Utilizing Thermopile Infrared Array Sensor. In: IEEE SENSORS 2014 Proceedings, 2014, pp. 462-465.

[5] Lin-jie Lu, Design and Implementation of Indoor Lighting Control System Based on Android (2013) In Chinese. 
[6] Kun Du, Lin Lv. The Design of DMX512 and STM32 Based Intelligent Interactive Lighting Control System. In: 2014 11th China International Forum on Solid State Lighting (SSLCHINA), Guangzhou, 2014, pp. 76-78.

[7] Tanaka, J; Shiozaki, M; Aita, F; Thermopile Infrared Array Sensor for Human Detector Application. In: 2014 IEEE 27th International Conference on Mocro Electro Mechanical Systems (MEMS), 2014, pp. 1213-1216.

[8] Hui Yao. The Design of a Led Stage Lighting Control System Based on DMX+ (2011) In Chinese.

[9] Shu. W. K, PBGA Wire Bonding Development. In: 1996 Proceedings 46th Electronic Components and Technology Conference, Orlando, FL, 1996, pp. 219-225.

[10]Ping Huang, Chun-hui Tang: Electronic Measurement Technology (2016).In Chinese. 